\title{
La dimensión sociocultural en los tests de inteligencia y logros
}

\author{
Julio Castillo
}

Toda cultura y todo grupo social tiene o ha tenido un sistema para jerarquizar la naturaleza de sus miembros. Esta jerarquización puede estar basada sobre la habilidad para cazar, la potencia sexual, la fuerza o la sabiduría. Todas estas dimensiones han servido al hombre como una forma de ordenamiento. Como una consecuencia de estas jerarquizaciones, estas dimensiones han dividido al hombre en diferentes grupos.

Esta práctica de ordenamiento también ocurre en nuestro tiempo. Es así que Jerome Kagan dice: "La sociedad americana contemporánea usa la inteligencia como una de las bases para jerarquizar a sus miembros..." (p. 53).

Pero este tipo de jerarquización (ranking) deja espacio para la especulación acerca de las diferencias en inteligencia entre los grupos humanos.

Durante el último siglo el argumento racista estuvo basado en la craneometría. Stephen Jay Gould dice: "Lo que la craneometría fue para el siglo XIX, han sido los tests de inteligencia para el siglo XX" (p. 146).

El argumento de este trabajo es que la construcción de tests de Coeficiente de Inteligencia (C.I.) y de logro tiene un "sesgo cultural" que provoca diferencias artificiales entre niños de diferente origen social, cultural o étnico, y los resultados de estos tests son debidos, en gran parte, a diferencias en el lenguaje, status socioeconómico y medio ambiente en el hogar, especialmente en aquellos tests relacionados con logro escolar.

De este argumento se desprenden dos objetivos principales:

1. Analizar cómo la construcción de tests, que son aplicados a niños de origen social, cultural y étnico diferente, tienen un "sesgo cultural" que produce diferencias artificiales entre ellos. 
2. Analizar el grado en el cual el lenguaje, el status socioeconómico y el medio ambiente en el hogar influyen en los resultados de estos tests entre los niños mencionados anteriormente.

Para explicar el sesgo cultural en la construcción de tests, es necesario desarrollar previamente un argumento clave.

\section{A. El argumento medioambiental}

Este argumento considera los efectos de factores medioambientales en las diferencias en el Coeficiente Intelectual. Al respecto, Sandra Scarr plantea que las diferencias raciales observadas en el C.I. están determinadas, básicamente, por factores medioambientales, los cuales producen fenotipos más pobres en circunstancias desventajosas, y no por diferencias genéticas sustanciales entre los grupos.

Scarr y Sapatek, en un estudio sobre gemelos humanos, comentado por la primera en su obra ya citada, han demostrado las predicciones de la hipótesis de desventajas medioambientales. En esta misma obra, cita otro estudio realizado junto a Weinberg, en el cual muestran que niños negros y de otros grupos interraciales, criados por familias blancas socioeconómicamente acomodadas, lograron puntajes más altos en un standard de C.I. y en tests escolares que otros niños de igual origen racial.

UN MODELO ALTERNATIVO PARA EL ANALISIS DE LAS DIFERENC1AS EN C.I. ENTRE GRUPOS

Peggy R. Sanday cita ejemplos de estudios sobre grupos que muestran puntajes en C.I. más bajos que los blancos de clase media y clase alta: niños de orfanatos (TYLER, 1965), niños montañeses (WHEELER, 1942), niños rurales (Tyler, 1965) y niños sordos (Graham y Shapiro, 1953). Tales grupos están aislados, en diferentes grados, de la corriente cultural americana principal de las clases media y alta. Sanday dice que los partidarios del enfoque de la herencia podrían argumentar que la inferioridad genética y la migración selectiva explica las diferencias en medias, entre grupos rurales, étnicos y de mayoría urbana. Sin embargo, ella argumenta una explicación alternativa, que es que estos grupos, en virtud de su aislamiento, no están expuestos a los elementos culturales relacionados con la expresión del C.I. La homogeneidad en la inteligencia medida entre estos grupos no se debe a una falta de intercambio o renovación genética, como podrían argumentar los partidarios del enfoque genetista. Se debe, más bien, a que estos grupos están expuestos a un medio ambiente uniforme en la ausencia de, al menos, algunos de los componentes relevantes que son importantes en las respuestas de los tests de C.I. Para Sanday (p. 239), la variación de C.I. dentro de los grupos es, generalmente, una función 
tanto de componentes genéticos como de componentes medioambientales. La expresión de la variación genética es una función de:

1. Cuidado médico pre y postnatal.

2. La dieta.

3. La estructura genética de los individuos.

La variación medioambiental es una función de:

1. Factores emocionales y motivaciones individuales; y

2. El grado y naturaleza del contacto con la cultura global.

Asumiendo que la distribución de la inteligencia, debida solamente a componentes genéticos, es similar en grupos raciales de una población y que las diferencias en puntajes de C.I. reportadas están relacionadas con una distribución desigual de los factores listados anteriormente, Sanday plantea su "hipótesis de difusión" (p. 241). Ella usa el término difusión en un sentido antropológico, esto es, el proceso por el cual los objetos materiales o las conductas aprendidas son transmitidas desde un punto de origen, dentro de una sociedad, a muchas otras sociedades. En este sentido, los miembros de diferentes grupos, a menudo, no comparten el mismo set de actos culturales y de componentes cognitivos basados en la cultura. Para Sanday, el proceso de difusión es considerado muy importante en la comprensión de las diferencias en el desempeño de tests entre grupos. Ella argumenta que un análisis de los ítem de tests de C.I. indica que muchos de ellos tienen información intencionada o sugerida. Esto implica que las diferencias significativas en respuestas correctas, entre grupos de la sociedad global y grupos minoritarios, podrían ocurrir en aquellos ítem que reflejan un contenido de la cultura global. De esta forma, la mayoría de los ítem de los tests presentan un sesgo cultural que produce diferencias en los resultados de grupos cultural y socialmente diferentes.

B. La CONSTRUCCión de TESTS Y SU APLiCACIÓN EN GRUPOS DE Distintos ORIGGENES: ÉTNICO, SOCIAL Y CULTURAL

La construcción de tests implica, al menos, dos dimensiones: lenguaje e información (contenido). Nosotros consideramos que el lenguaje está socialmente determinado y que la información es, en gran medida, un producto del medio ambiente sociocultural y de la experiencia personal. Se podría afirmar, por lo tanto, que la construcción de tests refleja el modelo de lenguaje y la información del grupo social al cual pertenece la persona que los confecciona. En este sentido, Jerome Kagan dice que la afirmación de que el ochenta por ciento de la inteligencia es hereditario y el veinte por ciento determinado por el medio ambiente, está basada en información proveniente de los tests 
de C.I. standarizados y similarmente construidos e inventados por personas occidentales de clase media caucásica, para clasificar a todo el mundo.

Kagan (p. 53) analiza el tipo de preguntas e ítem de tests, destacando las dificultades implicadas:

-El más importante set o conjunto de preguntas (importante, porque los puntajes sobre este conjunto tienen la más alta correlación con el C.I. total) solicita a la persona que defina palabras de rareza creciente. El dice que esta rareza es una cualidad relativa, dependiendo siempre del lenguaje de la comunidad que uno selecciona como referente.

El constructor de tests decidió que la rareza podría ser definida con respecto a la experiencia de la clase media caucásica.

Un segundo conjunto de preguntas plantea al niño algunas situaciones cotidianas y le pregunta qué habría hecho en esa situación. El problema aquí es que las preguntas suponen un medio ambiente de clase media urbana o suburbana. ¿Qué pasa con niños de medio ambiente rural de clase social baja?

-Una tercera clase de preguntas de test de C.I., llamadas analogías, tiene la misma validez dudosa que los tests de vocabulario, dado que los conceptos sobre los cuales el niño debe razonar tienen una familiaridad diferente para los varios grupos étnicos.

- La cuarta clase de preguntas pide a los niños resolver algunos problemas aritméticos. Por supuesto que si el niño no ha aprendido a sumar, restar o multiplicar, no estará en condiciones de resolverlos.

Otra clase de ítem incluye una línea dibujada de un objeto que tiene un elemento de menos y requiere que el niño descubra el rasgo que falta. Los dibujos están seleccionados para favorecer a los niños de clase media.

Kagan concluye que el test de C.I. es un instrumento seriamente sesgado, que casi garantiza puntajes más altos a los niños blancos de clase media que a los niños de otros grupos.

En Puerto Rico, tres tests de inteligencia -el de Stanford-Binet, el Wechsler Intelligence Scale for Children y el Goodenough Draw a Man Test - han sido traducidos y adaptados en el pasado, pero en todas las instancias, y debido a factores culturales, los investigadores han sido incapaces para redefinir los instrumentos suficientemente bien para obtener la media esperada de un C.I. de 100. Esto conduce a una subestimación de la inteligencia del niño portorriqueño. (Roca).

En otras palabras, los tests de C.I., en general, no toman en cuenta las diferencias culturales y sociales del medio ambiente donde los niños son socializados y el modo en que éste es percibido, provocando un sesgo cultural y social que produce diferencias artificiales, no reales, en el coeficiente intelectual de los niños. Como dice Erik Cohen: "La forma en que vemos nuestro medio ambiente depende ampliamente de lo que buscamos en él. Pero lo que buscamos no es un asunto individual o idiosincrático; depende de nuestro condiciona- 
miento cultural, de nuestros papeles sociales acostumbrados y de nuestra definición de la situación desde la cual nos relacionamos con el medio ambiente. Cualquier característica (un rio, un terreno estrecho o amplio, una montaña) puede ser vista desde una variedad de modos" (COHEN, p. 49).

Además del problema de la construcción de tests, hay otros que producen diferencias en los resultados de tests de C.I. y de logro entre grupos. A continuación serán analizadas las influencias del lenguaje, status socioeconómico y medio ambiente en el hogar, basados en los resultados de los tests entre diferentes grupos.

Herschel Manuel presenta el caso de niños hispanoparlantes del sudeste de Estados Unidos y las diferencias en habilidad y logro con niños angloparlantes en el mismo colegio o sistema educacional.

Un estudio citado por Manuel (p. 63), acerca de logro educacional en los grados 2 a 8 de un número de escuelas en el Valle Bajo de Río Grande (1930), dejó ver las serias condiciones promedio de alumnos hispanoparlantes y su baja reputación, año por año, en tests de lectura y aritmética. Una comparación de edades cronológicas mostró a los niños angloparlantes, en promedio, alrededor de un tercio de un año bajo las normas; pero los hispanoparlantes estaban, en promedio, alrededor de 3.6 años bajo las normas en lectura y alrededor de 2.6 años en aritmética. En otro estudio, Mary Anderson (1951) testeó habilidad y logro entre niños de primer grado, hispano y angloparlantes, usando el Cooperative Inter American Test of General Ability and Reading (en parte, un test no verbal). Ella encontró que nueve niños hispanoparlantes tuvieron puntajes no mejores que el promedio de los niños angloparlantes. Aplicando la Escala Warner para Status Socioeconómico, ella encontró que solamente 3 ó 4 por ciento de los niños hispanoparlantes estaban en los rangos medio y alto, en contraste con casi dos tercios de los niños angloparlantes.

Manuel (p. 65) cita el trabajo de Ivonne Ratliff, quien, usando el Test de Inteligencia de Goodenough en niños de primer grado, encontró que baja habilidad y bajo status socioeconómico están relacionados. El promedio de C.I. de niños hispano y angloparlantes fue de 93 y 107, respectivamente. Los hispanoparlantes fueron encontrados significativamente más bajos que los angloparlantes no sólo en logro escolar, sino también en status socioeconómico.

En otra investigación citada por Manuel (p. 66), el Test de Habilidad General Nivel 1, construido en ediciones paralelas inglesa y española, realizado en Colorado City bajo la supervisión de Helen K. Baily, mostró que más de un cuarto de los niños hispanoparlantes obtuvieron puntajes por sobre la media de los angloparlantes. Este mismo test fue aplicado en un colegio con una matrícula más o menos seleccionada de Ciudad de México, mostrando que la media de los niños hispanoparlantes fue más alta que la de los angloaparlantes.

Estos estudios muestran que el desarrollo mental de los niños en su primeros años está profundamente influenciado por el medio ambiente 
del hogar. Al mismo tiempo, la importancia del lenguaje es enfatizada también al descubrir un retardo progresivo de lectura en grados o cursos más avanzados.

Finalmente, Manuel (p. 69) dice que mucho de los niños hispanoparlantes vienen de hogares "culturalmente en desventaja". Muchos de ellos tiene poco o ningún conocimiento del lenguaje del colegio cuando se matriculan; muchos de ellos deben continuar su educación en un lenguaje diferente al del hogar; muchos de ellos carecen de un trasfondo de experiencia e incentivos que favorezcan un alto logro educacional. La falta de oportunidades comparables es una razón suficiente para sus bajos puntajes promedios en los tests. No hay ninguna razón para dudar de su capacidad básica de aprendizaje.

\section{Medio ambiente del hogar y Logro escolar}

La familia es una institución social, el lugar donde el niño experimenta su primer contacto con el mundo. En este medio, él aprende los hábitos, valores, normas, lenguajes, etc., de su grupo social, a través del proceso de socialización, de modo de llegar a ser un miembro de la sociedad. En otras palabras, él adquiere los elementos culturales de su grupo. Algunos autores hacen la distinción entre socialización primaria, realizada en el hogar, y socialización secundaria, desarrollada dentro del grupo de pares o en la escuela.

Sin duda que el tipo de medio ambiente creado por la familia va a tener una importante repercusión en el logro escolar y, antes que esto, en el desarrollo del niño durante sus primeros años. La importancia del medio ambiente del hogar en el desarrollo del niño ha sido destacada por muchos autores. Uno de ellos es Benjamín S. Bloom, quien plantea que en el hogar, especialmente entre los 2 y 10 años de edad, se desarrollan el lenguaje, la habilidad de aprender de los adultos y algunas de las cualidades de necesidad de logro, hábitos de trabajo y atención a las tareas, los cuales son básicos para el trabajo de los colegios. Desafortunadamente, no todos los hogares realizan esta labor del mismo modo y con los mismos resultados. El tiempo que los padres dedican a sus hijos, el modelo de lenguaje, la motivación del niño, el desarrollo del interés por aprender, etc., varían de un hogar a otro. Como resultado de esta variación, existen niños aventajados y desventajados, los cuales se desarrollan diferentemente en las escuelas. Tal como se dijo en la conferencia de Investigación sobre Deprivación Educacional y Cultural en la Universidad de Chicago, citada por Dave (p. 15): "Los objetos en el hogar, el grado de interés de los padres por enseñar y la cantidad de práctica y estimulación al niño a través de conversaciones y aprendizaje general, han sido encontradas como influencias significativas sobre el lenguaje y desarrollo cognitivo, desarrollo del interés por aprender, duración de la atención y motivación del niño". 
El mismo Dave analiza las influencias del medio ambiente educacional sobre los puntajes de logro escolar. El define el medio ambiente educacional como "aquellas condiciones, procesos y estímulos sociosicológicos del medio ambiente total, las cuales afectan el logro educacional del niño" (p. 16). El sostiene que este mèdio ambiente educacional puede estar presente en la escuela, en la sala de clases, en el hogar y en la comunidad; pero su estudio está enfocado hacia el medio ambiente del hogar que, en sus propias palabras, "...produce la primera $\mathrm{y}$, tal vez, la más insistente y sutil influencia en el desarrollo educacional del niño" (p. 117). El encontró que la correlación entre el indice del medio ambiente educacional y los puntajes totales de logro es .799 .

El trabajo de Dave y Wolf (Bloom, 1981) ha demostrado que "...lo que los padres hacen en el hogar, más que las características de su status, es un poderoso determinante del medio ambiente del hogar".

\section{Conclusiones}

En el intento de probar el argumento de este artículo, muchos puntos de vista acerca de las diferencias en el C.I. han sido revisados. Estos puntos de vista van desde el argumento medioambientalista hasta el argumento que da importancia a ambos factores: genético y medio ámbiente. Este último ayuda al argumento de este artículo en el sentido de que, utilizando un marco antropológico teórico, sostiene que las diferencias se deben, en gran medida, a trasfondos culturales disímiles.

La cultura del grupo determina, en una gran dimensión, el modo de pensar, la Weltanschauung de sus miembros, el modo de mirar al mundo y su medio ambiente. Luego, si este trasfondo cultural no es considerado cuando un test de C.I. o de logro es aplicado a diferentes grupos, los resultados mostrarán diferencias artificiales entre ellos. Este es el "sesgo cultural" que fue mencionado como parte del argumento. Ahora, si el mismo problema es analizado desde un punto de vista social, es posible argumentar que en estos tests podría haber un "sesgo social", aun cuando se testea a dos grupos con el mismo trasfondo cultural y perteneciendo a la misma sociedad. Todo esto, debido a que el conocimiento tiene una distribución social. Esto significa que los diferentes grupos, que forman parte de una sociedad, tienen diferentes conjuntos de conocimientos, como producto de su medio ambiente diferente y de un acceso diferencial a la información. Entonces es posible decir que los constructores de tests deben considerar estas diferencias.

Con respecto a las influencias del lenguaje, el status socioeconómico y el medio ambiente en el hogar, varias investigaciones han mostrado la importancia de estos factores en el logro escolar y, en general, en el desarrollo mental del niño. Dada la gran correlación 
entre el medio ambiente en el hogar, donde el desarrollo del lenguaje es uno de los rasgos básicos para el logro escolar, es necesario analizar cuidadosamente las diferencias en los resultados de tests entre niños que pertenecen a diferentes status socioeconómicos. En otras palabras, la presencia o ausencia de un medio ambiente educacional en el hogar, tal como fue definido por Dave, tendrá un gran efecto en los resultados de los tests.

Es posible que el C.I. esté influido por factores genéticos, pero también es cierto que un gran porcentaje de las diferencias de C.I. entre niños es debido a factores socioeconómicos, ambientales, así como a factores culturales.

\section{Bibliografía}

Bloom, B. S. (Ed.) Research Problems of Education and Cultural Deprivation. Chicago, University of Chicago, 1964. Human Characteristics and School Learning. Mac Graw-Hill, 1976. All Our Children Learning. Mac Graw-Hill, 1981.

Cohen, E.E. "Environmental Orientations: A Multidimensional Approach to Social Ecology", Current Anthropology, The University of Chicago Press, vol. $17, \mathrm{~N}^{\circ} 1$, march, 1976.

Dave, R. H. The Identification and Measurement of Environmental Process that are related to Educational Achievement. Chicago, University of Chicago, december, 1963.

Jay Gould, S. "Racist Argument and 1Q", en Race and IQ, A. Montagu (Ed.), Oxford, Oxford University Press, 1975.

Kagan, J. "The Magical Aura of the IQ", en Race and IQ, A. Montagu (Ed.), Oxford, Oxford University Press, 1975.

Manuel, Herschel. Spanish-Speaking Children of the Southwest. Their Education and the Public Welfare. Austin University of Texas Press, 1965.

Roca, P. Construction of a General Ability Group Test for Puerto Rican Students in the Elementary and Secondary Schools, Univesity of Puerto Rico, 1960.

Sanday, P. R. "On the Causes of IQ Differences Between Groups and Inıplications for Social Policy", en Race and IQ, A. Montagu (Ed.), Oxford, Oxford University Press, 1975.

Scarr, S. Race, Social Class, and Individual Differences, en Race and IQ. New Jersey, Hilldale, 1981. 